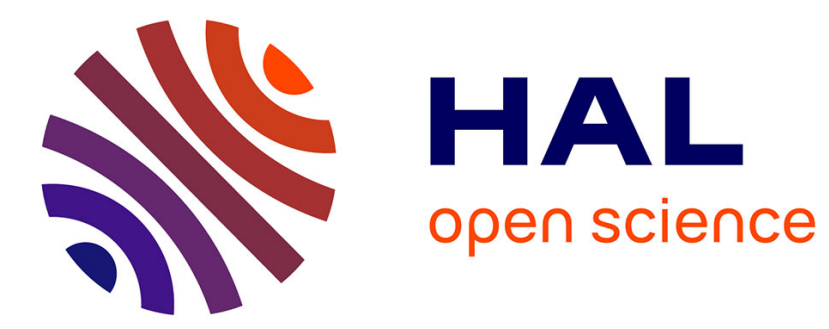

\title{
A cracked beam finite element for through-cracked tube
} Franck Schoefs, Anh Le Van, Mustapha Rguig

\section{To cite this version:}

Franck Schoefs, Anh Le Van, Mustapha Rguig. A cracked beam finite element for throughcracked tube. Communications in Numerical Methods in Engineering, 2007, 24 (9), pp.761 - 775. 10.1002/cnm.988 . hal-01004696

\section{HAL Id: hal-01004696 https://hal.science/hal-01004696}

Submitted on 22 Oct 2016

HAL is a multi-disciplinary open access archive for the deposit and dissemination of scientific research documents, whether they are published or not. The documents may come from teaching and research institutions in France or abroad, or from public or private research centers.
L'archive ouverte pluridisciplinaire HAL, est destinée au dépôt et à la diffusion de documents scientifiques de niveau recherche, publiés ou non, émanant des établissements d'enseignement et de recherche français ou étrangers, des laboratoires publics ou privés. 


\title{
A cracked beam finite element for through-cracked tube
}

\author{
F. Schoefs, A. Le Van and M. Rguig \\ Institute of Civil and Mechanical Research (GeM), UMR-CNRS 6183, Nantes Atlantic University, 2 rue de la \\ Houssinière, B.P. 9220844322 Nantes Cedex 03, France
}

\begin{abstract}
SUMMARY
The reassessment of existing structures leads to analyse the impact of local defects on the structural behaviour. This paper proposes a cracked beam finite element with a view to introducing the effect of large through-cracks in the structural analysis for framed structures like jacket offshore platforms. The model parameters are identified for several joint typologies using 3D finite element results.
\end{abstract}

KEY WORDS: cracked beam finite element; through-crack; displacement method; offshore structures

\section{INTRODUCTION}

The study of the impact of through-cracks on the structural integrity of offshore structures such as jacket platforms is still a challenge. For reassessment purposes, one needs to develop specific criteria so as to introduce the effect of structural redundancy in presence of a through-crack and decide whether to repair or not. The purpose of this paper is to build a finite element representing the behaviour of a through-cracked tube. The paper depicts how to transfer the main mechanical effects of a through-crack to the structure behaviour using an equivalent cracked beam finite element (CBFE). A number of studies, e.g. [1-5], have been carried out, yet giving rather incomplete solutions mainly for structural components loaded in their plane. Some were devoted to the influence of the joint flexibility in offshore structures subjected to fatigue loadings; see, for instance, [6-8]. The formulations proposed therein showed the significant role of the loadings as well as the typologies of nodes (T-joint, Y-joint, K-joint or TK-joint). In [7], the flexible joints were modelled by means of three springs and their stiffnesses were given in parametric forms. 
Other authors modelled complex joints without cracks by using thin shell elements, e.g. $[9,10]$; or to introduce specific limit conditions in buckling analysis [4].

The CBFE proposed in this paper enables one to consider all geometrical typologies of nodes which are encountered on jacket structures. First, we describe the cracked beam by introducing four mechanical parameters (two stiffnesses and two eccentricities). The stiffness matrix is derived from the strain energy as a function of the four parameters. Also, the comparison is made with the force method. Second, the model parameters are identified using 3D finite element results. The efficiency of the proposed model is shown through some specific joint typologies.

\section{DESCRIPTION OF THE CRACKED BEAM FINITE ELEMENT (CBFE)}

This section describes the CBFE with the purpose analysing the behaviour of a through-cracked tube, see Figure 1. The through-crack is modelled as a loss of stiffness which is represented by two torsional springs and two eccentricities due to the shift of neutral axis of the tube. Therefore, applying an axial force leads to a momentum at the cracked section. This effect is significant when analysing framed structures such as jackets offshore platforms.

\subsection{The stiffness matrix $K$ for a cracked tube}

The cracked finite element will be next described in the local axis system. The $x$-axis is directed towards the tube length (Figure 2) whereas the $y$ and $z$-axes can be arbitrarily chosen (since the cross-section is circular, any axis in the section is principal). The shift of the neutral axis along the $y$ and $z$-axes is represented by two eccentricities $e^{y}$ and $e^{z}$ and the stiffness loss by two torsional springs of stiffness $k^{y}$ and $k^{z}$ between points 1 and 4 .

Let us denote by $U$ the nodal generalized displacement vector of the cracked beam element, including rotations $\theta_{4}^{y}$ and $\theta_{4}^{z}$ at node 4 about $y$ and $z$ axes, respectively (Figure 2). The degrees of freedom $\theta_{4}^{y}$ and $\theta_{4}^{z}$ are added to the standard displacement vector of displacement in order to take into account the fact that they are variables independent to others

$$
U^{\mathrm{T}}=\left\langle\begin{array}{llllllllllllll}
u_{1} & v_{1} & w_{1} & \theta_{1}^{x} & \theta_{1}^{y} & \theta_{1}^{z} & u_{2} & v_{2} & w_{2} & \theta_{2}^{x} & \theta_{2}^{y} & \theta_{2}^{z} & \theta_{4}^{y} & \theta_{4}^{z}
\end{array}\right\rangle
$$

where $u_{i}, v_{i}, w_{i}$ are the nodal displacements and $\theta_{i}^{j}$ the nodal rotations.

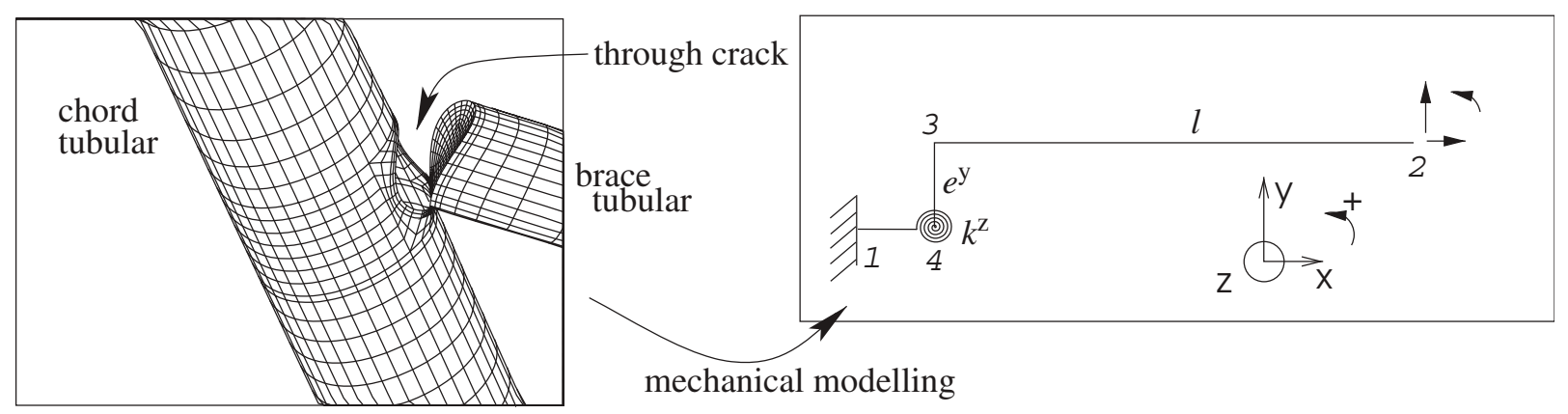

Figure 1. Mechanical model for a cracked tube in plane $(x y)$ [2]. 


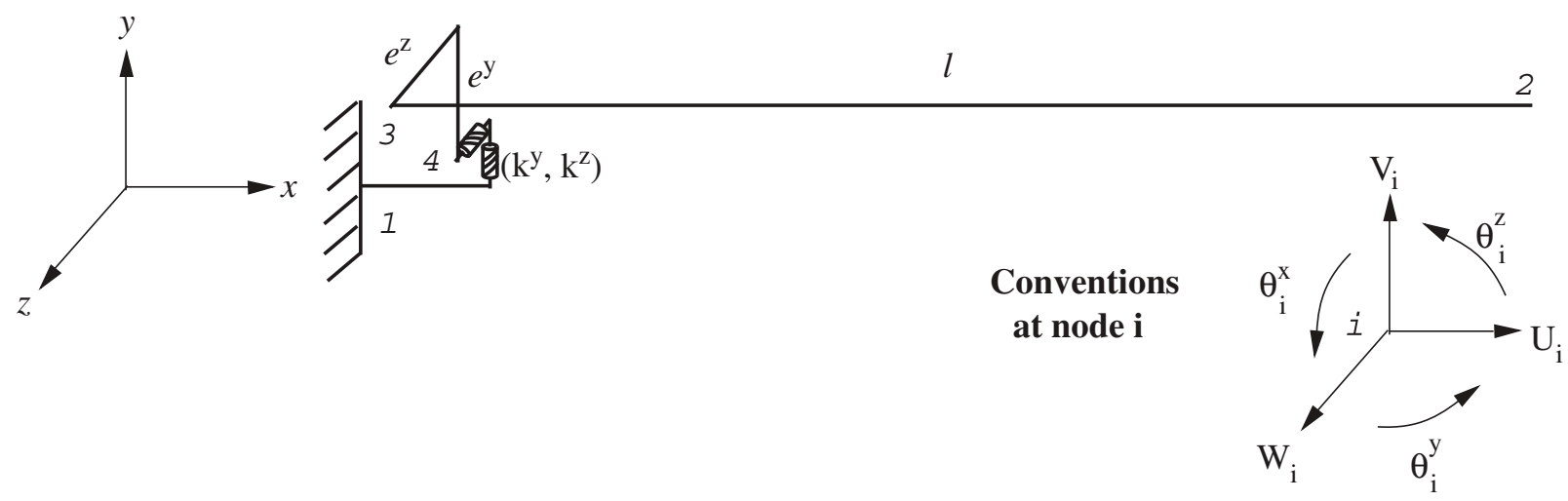

Figure 2. The cracked beam finite element (CBFE) in 3D space.

The strain energy of the cracked tube is

$$
\begin{aligned}
\mathcal{W}= & \frac{1}{2} \int_{0}^{l} E S\left(\frac{\mathrm{d} u(x)}{\mathrm{d} x}\right)^{2} \mathrm{~d} x+\frac{1}{2} \int_{0}^{l} E I\left(\frac{\mathrm{d}^{2} v(x)}{\mathrm{d} x^{2}}\right)^{2} \mathrm{~d} x+\frac{1}{2} \int_{0}^{l} E I\left(\frac{\mathrm{d}^{2} w(x)}{\mathrm{d} x^{2}}\right)^{2} \mathrm{~d} x \\
& +\frac{1}{2} \int_{0}^{l} G J\left(\frac{\mathrm{d} \theta^{x}(x)}{\mathrm{d} x}\right)^{2} \mathrm{~d} x+\frac{1}{2} k^{y}\left(\theta_{1}^{z}-\theta_{4}^{z}\right)^{2}+\frac{1}{2} k^{z}\left(\theta_{1}^{y}-\theta_{4}^{y}\right)^{2}
\end{aligned}
$$

where $S$ denotes the cross-sectional area, $I$ the bending second moment of area, $J$ the torsional second moment of area, $E$ the Young modulus and $G$ the shear modulus.

The displacements $u(x), v(x), w(x)$ and the twist rotation $\theta^{x}(x)$ between points 3 and 2 are interpolated in a usual way

$$
\begin{aligned}
u(x)= & \frac{x}{l} u_{2}+\left(1-\frac{x}{l}\right) u_{3} \\
v(x)= & \left(1-\frac{3}{l^{2}} x^{2}+\frac{2}{l^{3}} x^{3}\right) v_{3}+\left(x-\frac{2}{l} x^{2}+\frac{1}{l^{2}} x^{3}\right) \theta_{3}^{z} \\
& +\left(\frac{3}{l^{2}} x^{2}-\frac{2}{l^{3}} x^{3}\right) v_{2}+\left(\frac{1}{l^{2}} x^{3}-\frac{1}{l} x^{2}\right) \theta_{2}^{z} \\
w(x)= & \left(1-\frac{3}{l^{2}} x^{2}+\frac{2}{l^{3}} x^{3}\right) w_{3}-\left(x-\frac{2}{l} x^{2}+\frac{1}{l^{2}} x^{3}\right) \theta_{3}^{y} \\
& +\left(\frac{3}{l^{2}} x^{2}-\frac{2}{l^{3}} x^{3}\right) w_{2}-\left(\frac{1}{l^{2}} x^{3}-\frac{1}{l} x^{2}\right) \theta_{2}^{y} \\
\theta^{x}(x)= & \left(1-\frac{x}{l}\right) \theta_{3}^{x}+\frac{x}{l} \theta_{2}^{x}
\end{aligned}
$$


As a matter of fact, points 1 and 4 are at the same position. Hence,

$$
\begin{aligned}
u_{1} & =u_{4} \\
v_{1} & =v_{4} \\
w_{1} & =w_{4} \\
\theta_{1}^{x} & =\theta_{4}^{x}
\end{aligned}
$$

The portion of the beam element between points 3 and 4 , which represents the eccentricities, is considered as rigid. Therefore,

$$
\begin{aligned}
& \theta_{3}^{x}=\theta_{4}^{x} \\
& \theta_{3}^{y}=\theta_{4}^{y} \\
& \theta_{3}^{z}=\theta_{4}^{z}
\end{aligned}
$$

By assuming small rotations in portions $4-3$, the displacement of point 3 is related to that of point 4 by the rigid solid relationship

$$
\mathbf{U}_{3}=\mathbf{U}_{4}+\boldsymbol{\theta}_{34} \wedge \mathbf{L}_{43}
$$

where $\mathbf{U}_{3}=\left\langle u_{3}, v_{3}, w_{3}\right\rangle$ is the displacement of node $3, \mathbf{U}_{4}=\left\langle u_{4}, v_{4}, w_{4}\right\rangle$ the displacement of node $4, \boldsymbol{\theta}_{34}=\left\langle\theta_{3}^{x}, \theta_{3}^{y}, \theta_{3}^{z}\right\rangle$ the rotation vector of the rigid element $3-4$ and $\mathbf{L}_{43}=\left\langle 0, e^{y}, e^{z}\right\rangle$ the vector relating nodes 3 and 4 .

Inserting conditions (4) and (5) into Equation (6) leads to

$$
\begin{aligned}
& u_{3}=u_{1}+e^{z} \theta_{4}^{y}-e^{y} \theta_{4}^{z} \\
& v_{3}=v_{1}-e^{z} \theta_{1}^{x} \\
& w_{3}=w_{1}+e^{y} \theta_{1}^{x}
\end{aligned}
$$

Relations (3) and (7) give rise to expressions for the terms in the strain energy (2)

$$
\begin{aligned}
\frac{\mathrm{d} u(x)}{\mathrm{d} x} & =N_{1}^{\mathrm{T}}(x) U \\
\frac{\mathrm{d}^{2} v(x)}{\mathrm{d} x^{2}} & =N_{2}^{\mathrm{T}}(x) U \\
\frac{\mathrm{d}^{2} w(x)}{\mathrm{d} x^{2}} & =N_{3}^{\mathrm{T}}(x) U \\
\frac{\mathrm{d} \theta^{x}(x)}{\mathrm{d} x} & =N_{4}^{\mathrm{T}}(x) U \\
\theta_{1}^{y}-\theta_{4}^{y} & =N_{5}^{\mathrm{T}}(x) U \\
\theta_{1}^{z}-\theta_{4}^{z} & =N_{6}^{\mathrm{T}}(x) U
\end{aligned}
$$


where vectors $N_{i}(x)$ are defined as

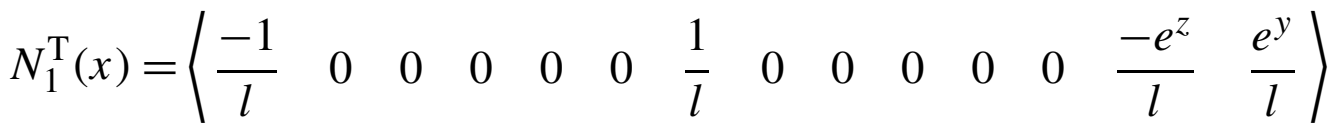

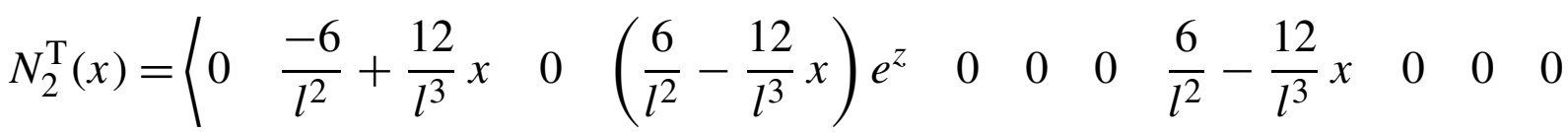

$$
\begin{aligned}
& \left.\frac{6}{l^{2}} x-\frac{2}{l} \quad 0 \quad \frac{-4}{l}+\frac{6}{l^{2}} x\right\rangle
\end{aligned}
$$

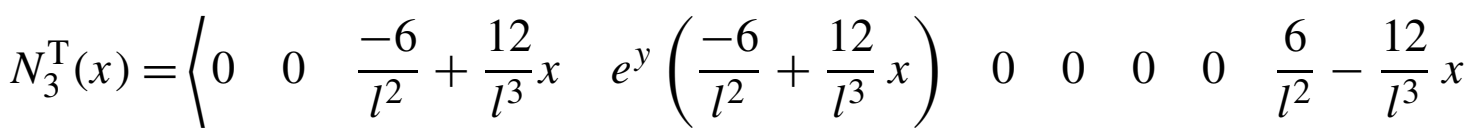

$$
\begin{aligned}
& \left.0 \quad \frac{-6}{l^{2}} x+\frac{2}{l} \quad 0 \quad \frac{4}{l}-\frac{6}{l^{2}} x \quad 0\right\rangle \\
& N_{4}^{\mathrm{T}}(x)=\left\langle\begin{array}{llllllllllllll}
0 & 0 & 0 & \frac{-1}{l} & 0 & 0 & 0 & 0 & 0 & \frac{1}{l} & 0 & 0 & 0 & 0
\end{array}\right\rangle \\
& N_{5}^{\mathrm{T}}(x)=\left\langle\begin{array}{llllllllllllll}
0 & 0 & 0 & 0 & 1 & 0 & 0 & 0 & 0 & 0 & 0 & 0 & -1 & 0
\end{array}\right\rangle \\
& N_{6}^{\mathrm{T}}(x)=\left\langle\begin{array}{llllllllllllll}
0 & 0 & 0 & 0 & 0 & 1 & 0 & 0 & 0 & 0 & 0 & 0 & 0 & -1
\end{array}\right\rangle
\end{aligned}
$$

Thus, the strain energy (2) can be expressed as a function of the nodal displacement vector $U$

$$
\begin{aligned}
\mathcal{W}= & \frac{1}{2} U^{\mathrm{T}}\left(\int_{0}^{l} E S N_{1}(x) N_{1}^{\mathrm{T}}(x) \mathrm{d} x+\int_{0}^{l} E I N_{2}(x) N_{2}^{\mathrm{T}}(x) \mathrm{d} x+\int_{0}^{l} E I N_{3}(x) N_{3}^{\mathrm{T}}(x) \mathrm{d} x\right. \\
& \left.+\int_{0}^{l} G J N_{4}(x) N_{4}^{\mathrm{T}}(x) \mathrm{d} x+k^{y} N_{5}(x) N_{5}^{\mathrm{T}}(x)+k^{z} N_{6}(x) N_{6}^{\mathrm{T}}(x)\right) U
\end{aligned}
$$

The stiffness matrix $K$ is then derived from its definition $\mathcal{W}=\frac{1}{2} U^{\mathrm{T}} K U$ as

$$
\begin{aligned}
K= & \int_{0}^{l} E S N_{1}(x) N_{1}^{\mathrm{T}}(x) \mathrm{d} x+\int_{0}^{l} E I N_{2}(x) N_{2}^{\mathrm{T}}(x) \mathrm{d} x+\int_{0}^{l} E I N_{3}(x) N_{3}^{\mathrm{T}}(x) \mathrm{d} x \\
& +\int_{0}^{l} G J N_{4}(x) N_{4}^{\mathrm{T}}(x) \mathrm{d} x+k^{y} N_{5}(x) N_{5}^{\mathrm{T}}(x)+k^{z} N_{6}(x) N_{6}^{\mathrm{T}}(x)
\end{aligned}
$$

Due to the additional degrees of freedom $\theta_{4}^{y}$ and $\theta_{4}^{z}$, the stiffness matrix is of dimension $14 \times 14$ instead of the usual dimension $12 \times 12$. The stiffness matrix $K$ can be recast as follows:

$$
K=\left(\begin{array}{lll}
K_{11} & K_{12} & K_{1 a} \\
K_{21} & K_{22} & K_{2 a} \\
K_{a 1} & K_{a 2} & K_{a a}
\end{array}\right)
$$


where the subscript $a$ recalls the additional degrees of freedom $\theta_{4}^{y}$ and $\theta_{4}^{z}$ and

$$
K_{11}=\left(\begin{array}{cccccc}
\frac{E S}{l} & 0 & 0 & 0 & 0 & 0 \\
0 & \frac{12 E I}{l^{3}} & 0 & -e^{z} \frac{12 E I}{l^{3}} & 0 & 0 \\
0 & 0 & \frac{12 E I}{l^{3}} & e^{y} \frac{12 E I}{l^{3}} & 0 & 0 \\
0 & -e^{z} \frac{12 E I}{l^{3}} & e^{y} \frac{12 E I}{l^{3}} & \frac{G J}{l}+\left(e^{y 2}+e^{z 2}\right) \frac{12 E I}{l^{3}} & 0 & 0 \\
0 & 0 & 0 & 0 & k^{y} & 0 \\
0 & 0 & 0 & 0 & 0 & k^{z}
\end{array}\right)
$$$$
K_{12}=K_{21}^{\mathrm{T}}=\left(\begin{array}{cccccc}
\frac{-E S}{l} & 0 & 0 & 0 & 0 & 0 \\
0 & \frac{-12 E I}{l^{3}} & 0 & 0 & 0 & \frac{6 E I}{l^{2}} \\
0 & 0 & \frac{-12 E I}{l^{3}} & 0 & \frac{-6 E I}{l^{2}} & 0 \\
0 & e^{z} \frac{12 E I}{l^{3}} & -e^{y} \frac{12 E I}{l^{3}} & \frac{-G J}{l} & -e^{y} \frac{6 E I}{l^{2}} & -e^{z} \frac{6 E I}{l^{2}} \\
0 & 0 & 0 & 0 & 0 & 0 \\
0 & 0 & 0 & 0 & 0 & 0
\end{array}\right)
$$

$$
\begin{aligned}
& K_{22}=\left(\begin{array}{cccccc}
\frac{E S}{l} & 0 & 0 & 0 & 0 & 0 \\
0 & \frac{12 E I}{l^{3}} & 0 & 0 & 0 & \frac{-6 E I}{l^{2}} \\
0 & 0 & \frac{12 E I}{l^{3}} & 0 & \frac{6 E I}{l^{2}} & 0 \\
0 & 0 & 0 & \frac{G J}{l} & 0 & 0 \\
0 & 0 & \frac{6 E I}{l^{2}} & 0 & \frac{4 E I}{l} & 0 \\
0 & \frac{-6 E I}{l^{2}} & 0 & 0 & 0 & \frac{4 E I}{l}
\end{array}\right) \\
& K_{1 a}^{\mathrm{T}}=K_{a 1}=\left(\begin{array}{cccccc}
e^{z} \frac{E S}{l} & 0 & \frac{-6 E I}{l^{2}} & -e^{y} \frac{6 E I}{l^{2}} & -k^{y} & 0 \\
-e^{y} \frac{E S}{l} & \frac{6 E I}{l^{2}} & 0 & -e^{z} \frac{6 E I}{l^{2}} & 0 & -k^{z}
\end{array}\right)
\end{aligned}
$$




$$
\begin{aligned}
K_{2 a}^{\mathrm{T}}=K_{a 2}= & \left(\begin{array}{cccccc}
-e^{z} \frac{E S}{l} & 0 & \frac{6 E I}{l^{2}} & 0 & \frac{2 E I}{l} & 0 \\
e^{y} \frac{E S}{l} & \frac{-6 E I}{l^{2}} & 0 & 0 & 0 & \frac{2 E I}{l}
\end{array}\right) \\
K_{a a}= & \left(\begin{array}{ccc}
k^{y}+e^{z 2} \frac{E S}{l}+\frac{4 E I}{l} & -e^{y} e^{z} \frac{E S}{l} \\
-e^{y} e^{z} \frac{E S}{l} & k^{z}+e^{y 2} \frac{E S}{l}+\frac{4 E I}{l}
\end{array}\right)
\end{aligned}
$$

If the crack does not exist, the eccentricities $e^{y}$ and $e^{z}$ tend to 0 whereas the torsional stiffnesses tend to infinity. The points 1,3 and 4 coincide so that one can sum up the lines and columns corresponding to $\theta_{1}^{y}$ and $\theta_{4}^{y}$ and those corresponding to $\theta_{1}^{z}$ and $\theta_{4}^{z}$. As expected, the stiffness matrix of the cracked finite element then reduces to the well-known uncracked beam element.

The equilibrium equation for a CBFE is

$$
K U=F \quad \text { or } \quad\left(\begin{array}{lll}
K_{11} & K_{12} & K_{1 a} \\
K_{21} & K_{22} & K_{2 a} \\
K_{a 1} & K_{a 2} & K_{a a}
\end{array}\right)\left(\begin{array}{c}
U_{1} \\
U_{2} \\
U_{a}
\end{array}\right)=\left(\begin{array}{c}
F_{1} \\
F_{2} \\
0
\end{array}\right)
$$

where $U_{a}^{\mathrm{T}}=\left\langle\theta_{4}^{y} \quad \theta_{4}^{z}\right\rangle$ and $F_{1}, F_{2}$ are loading vectors, respectively, at nodes 1 and 2.

\subsection{Comparison with the force method}

The force method was described in [1,2]. This method consists in computing the compliance matrix for a cracked finite element from the stress energy. The stiffness matrix $K$ is then obtained as the inverse of the compliance matrix. Its expression was given in [1] for the 2D case. In order to compare the force method with the CBFE model proposed herein, we have extended the aforementioned expression to the 3D case. The symbolic calculation, conducted using the Matlab software [11], was lengthy and the resulting expression for $K$ was much more complex than (12)-(18). Let us here give only the term $K(1,1)$ obtained from the force method

$$
K(1,1)=\frac{f_{1} E S}{l f_{2}}
$$

with

$$
\begin{aligned}
& f_{1}=\left(4 E I+l k^{y}\right)\left(4 E I+l k^{z}\right) \\
& f_{2}=4 E I\left(4 E I+l k^{y}+l k^{z}\right)+4 E I E S\left(e^{y 2}+e^{z 2}\right)+l E S\left(e^{y 2} k^{y}+e^{z 2} k^{z}\right)+l^{2} k^{y} k^{z}
\end{aligned}
$$

compared to expression for $K(1,1)=E S / l$ in (13).

However, further tedious computations show that by and large the force method and the CBFE model do give the same final results, as expected. This has been checked on the example of a T-joint structure composed by a vertical beam clamped at its ends and a horizontal cracked beam welded onto the vertical beam and loaded at the free end. The parameters $e^{y}, e^{z}, k^{y}$ and $k^{z}$ are identified following the procedure described in Section 3. It is found that the strain (or stress) energy obtained by the two methods are identical to within less than $0.1 \%$. In the following, only the stiffness matrix obtained by the CBFE model is considered. 


\section{IDENTIFICATION OF MODEL PARAMETERS}

\subsection{Identification principle}

The cracked finite element involves the parameters $e^{y}, e^{z}, k^{y}$ and $k^{z}$ which are to be identified. The identification will be carried out on the problem of a cracked tube built in a rigid plate, see Figure 3 where the local axis systems relative to the cracked tube and the plate are shown. The $y_{0}$-axis for the plate is directed along by the intersection line between the plane of the cracked section and the symmetry plane $(x, y)$ of the structure. The dimensions of the tube are given standard values in offshore engineering: length $l=10.2 \mathrm{~m}$, average radius $r_{\mathrm{m}}=0.55 \mathrm{~m}$ and thickness $t=0.02 \mathrm{~m}$. On the other hand, the parameter identification is performed with various positions of the tube and the crack defined by: the angle $\lambda$ between $x$ and $y_{0}$-axes; the angle $\psi$ between the crack axis and $y_{0}$-axis; and the half-opening angle $\varphi$ of the crack.

Given a triplet $(\lambda, \psi, \varphi)$, the parameters $e^{y}, e^{z}, k^{y}$ and $k^{z}$ are identified by minimizing-in the least-squares sense-the difference $Q$ between the displacement $U_{2}$ at the free end of the CBFE model and the displacement $\tilde{U}_{2}$ obtained from 3D finite element computations.

$$
\min Q=\min \left\|U_{2}-\tilde{U}_{2}\right\|^{2}
$$

\section{2. $3 D$ finite element computations}

For each triplet $(\lambda, \psi, \varphi)$, the target displacement $\tilde{U}$ for the minimization problem (23) is obtained from 3D finite element computations using the software CAST3M [12]. A specific routine is added in CAS3TM in order to generate regular meshes in the neighbourhood of the crack front (Figure 4(a)). The tube mesh is composed of 20-node brick elements, with nel elements along the tube length and nec elements along the circumference of the cross-section.

\section{Remark}

Other meshes with 8-node rectangular shell elements are also used, see Figure 4(b). In fact, whereas the 3D and shell elements give the same global results, only the 3D meshes allow to the stress intensity factors, which are not presented in this work, to be accurately computed. Therefore, only the numerical results obtained with $3 \mathrm{D}$ elements are presented in this paper.
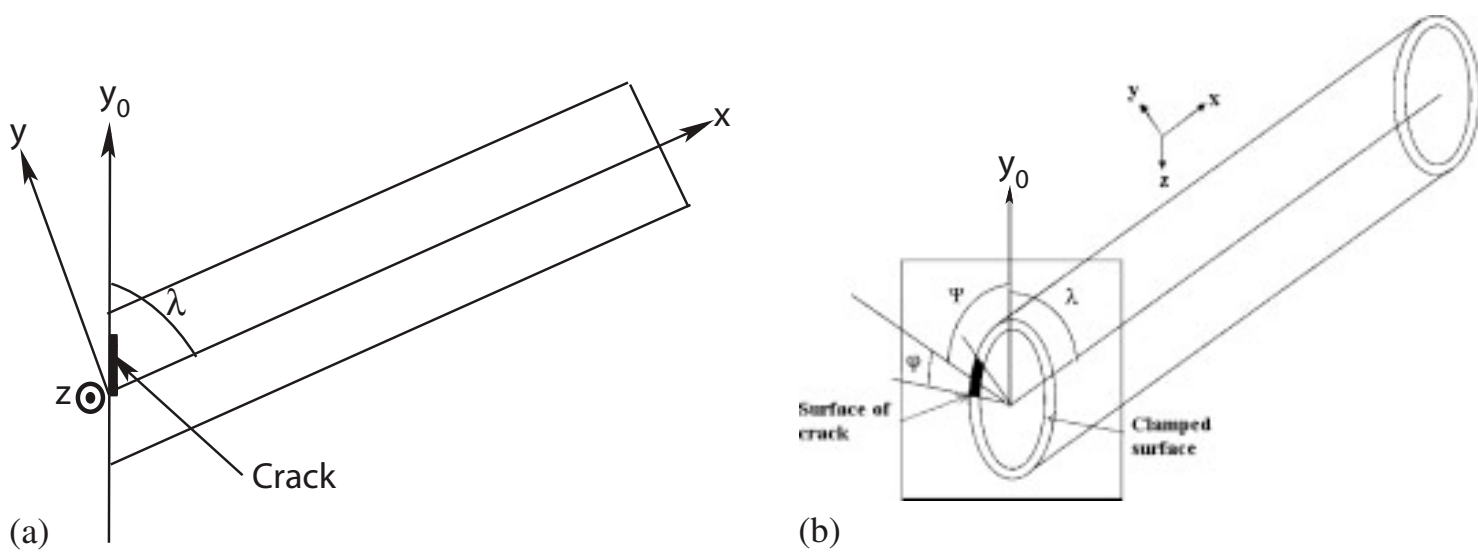

Figure 3. Geometry of the cracked tube. 


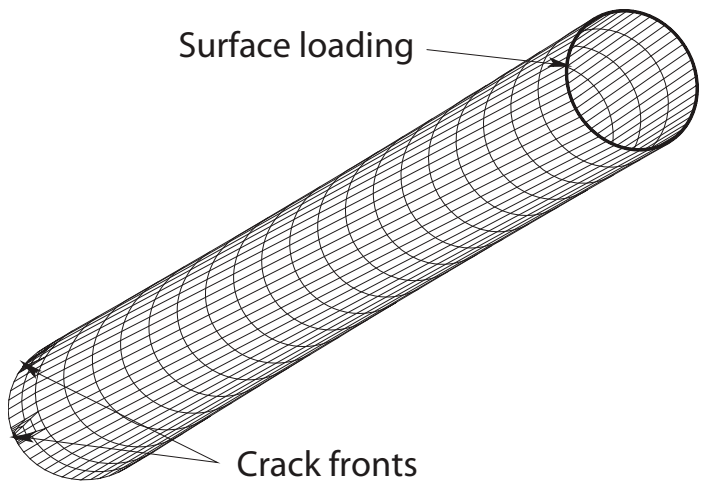

(a)

(b)

Figure 4. Meshes of the cracked tube: (a) 3D FE mesh of the cracked tube $\left(\lambda=45^{\circ}, \psi=45^{\circ}, \varphi=40^{\circ}\right)$ and (b) FE mesh using shell elements $\left(\lambda=45^{\circ}, \psi=150^{\circ}, \varphi=30^{\circ}\right)$.

\subsection{Formulas for identifying the model parameters}

The displacement $U_{2}$ in (23) can be expressed in terms of the model parameters by solving Equation (19) with $u_{1}=0$

$$
U_{2}=S_{2} F_{2}, \quad S_{2}=\left[K_{22}-K_{2 a} K_{a a}^{-1} K_{a 2}\right]^{-1}
$$

where $S_{2}$ denotes the compliance matrix condensed at node 2 of the cracked finite element. In the sequel, in order to facilitate the computations, all the equations will be written using rather the homogenized displacement vector $\tilde{U}_{2}^{*}$ obtained by multiplying the rotations by the length $l$ of the element, and the homogenized force vector $F_{2}^{*}$ by dividing the moments by $l$. With these homogenized variables, the minimization problem reads

$$
\min Q^{*}=\min \left\|S_{2}^{*} F_{2}^{*}-\tilde{U}_{2}^{*}\right\|^{2}
$$

where $S_{2}^{*}$ are the homogenized compliance matrix.

For brevity, $\widetilde{U}_{2}^{*}, F_{2}^{*}, S_{2}^{*}$ will be denoted $\widetilde{U}^{*}, F^{*}, S^{*}$ in the sequel. By noting that

$$
S^{* \mathrm{~T}}=S^{*} \Rightarrow\left(\frac{\partial S^{*}}{\partial e_{y}}\right)^{\mathrm{T}}=\frac{\partial S^{*}}{\partial e_{y}}
$$


it can be shown that minimizing $Q$ with respect to $e^{y}, e^{z}, k^{y}$ and $k^{z}$ leads to the following equation system:

$$
\begin{aligned}
& F^{* \mathrm{~T}}\left[S^{*} \frac{\partial S^{*}}{\partial e^{y}}+\frac{\partial S^{*}}{\partial e^{y}} S^{*}\right] F^{*}-2 \tilde{U}^{* \mathrm{~T}}\left[\frac{\partial S^{*}}{\partial e^{y}}\right] F^{*}=0 \\
& F^{* \mathrm{~T}}\left[S^{*} \frac{\partial S^{*}}{\partial e^{z}}+\frac{\partial S^{*}}{\partial e^{z}} S^{*}\right] F^{*}-2 \tilde{U}^{* \mathrm{~T}}\left[\frac{\partial S^{*}}{\partial e^{z}}\right] F^{*}=0 \\
& F^{* \mathrm{~T}}\left[S^{*} \frac{\partial S^{*}}{\partial k^{y}}+\frac{\partial S^{*}}{\partial k^{y}} S^{*}\right] F^{*}-2 \tilde{U}^{* \mathrm{~T}}\left[\frac{\partial S^{*}}{\partial k^{y}}\right] F^{*}=0 \\
& F^{* \mathrm{~T}}\left[S^{*} \frac{\partial S^{*}}{\partial k^{z}}+\frac{\partial S^{*}}{\partial k^{z}} S^{*}\right] F^{*}-2 \tilde{U}^{* \mathrm{~T}}\left[\frac{\partial S^{*}}{\partial k^{z}}\right] F^{*}=0
\end{aligned}
$$

It has been shown in [2] that applying a shear force or a bending moment at the free end of the element leads to same results for the minimization problem. Here, we choose to apply a shear force $T^{y}$ along the $y$-axis so as to identify $e^{y}$ and $k^{z}$. The load vector is then $\left.F^{* T}=\begin{array}{llllll}0 & T^{y} & 0 & 0 & 0 & 0\end{array}\right)$. Solving Equation (27) then yields

$$
\begin{aligned}
e_{y} & =l \frac{12 \widetilde{u}}{5 T^{y} l^{3} / E I-6\left(\widetilde{v}+\widetilde{\theta}^{z}\right)} \\
k_{z} & =\frac{E I}{l} \frac{12 T^{y} l^{3} / E I}{6\left(\widetilde{v}+l \widetilde{\theta}^{z}\right)-5 T^{y} l^{3} / E I}
\end{aligned}
$$

In order to identify $e^{z}$ and $k^{y}$, let us now apply a shear force $T^{z}$ along the $z$-axis, i.e. $F^{* \mathrm{~T}}=\left\langle\begin{array}{llllll}0 & 0 & T^{z} & 0 & 0 & 0\end{array}\right\rangle$. Solving Equation (27) then yields

$$
\begin{aligned}
& e_{z}=l \frac{12 \widetilde{u}}{5 T^{z} l^{3} / E I-6\left(\widetilde{w}-l \widetilde{\theta}^{y}\right)} \\
& k_{y}=\frac{E I}{l} \frac{12 T^{z} l^{3} / E I}{6\left(\widetilde{w}-l \widetilde{\theta}^{y}\right)-5 T^{z} l^{3} / E I}
\end{aligned}
$$

The study of the convergence of the CBFE model parameters is carried out with respect to the mesh refinement, namely the element numbers nel and nec. Figures 5(a) and (b) plot eccentricity $e^{y}$ versus nel (nel $\left.=30-50\right)$ and nec $($ nec $=30-200)$ for $\lambda=90^{\circ}, \psi=0^{\circ}$ and $\varphi=16^{\circ}$. It is found that $e^{y}$ varies little with nel-the variation is less than 3\%-whereas it varies significantly with nec. The parameter identification is highly sensitive to the robustness of the $3 \mathrm{D}$ meshes and to the assessment of the homogenized displacement at the end of the beam. In case of throughcracks the computation of $\tilde{u}$ is very sensitive to the mesh. This is mainly due to the sensitivity to the longitudinal displacement at the free end of the finite element. Hence, in practical use, the above expressions for $e^{y}$ and $e^{z}$ can be advantageously replaced with the available semianalytical ones. Without going into details, here we give the semi-analytical formulas for $e^{y}$ and $e^{z}$ 

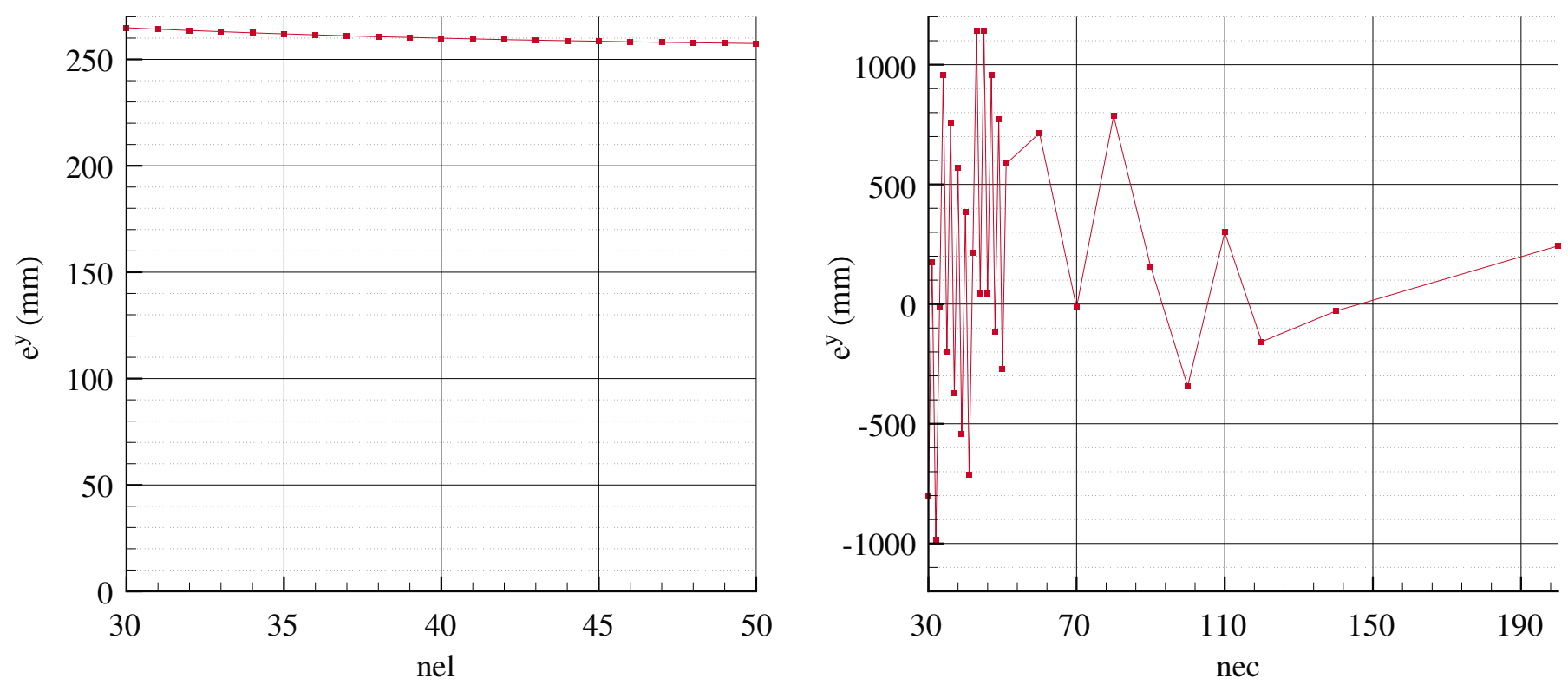

Figure 5. Eccentricity $e^{y}$ versus element numbers nel and nec.
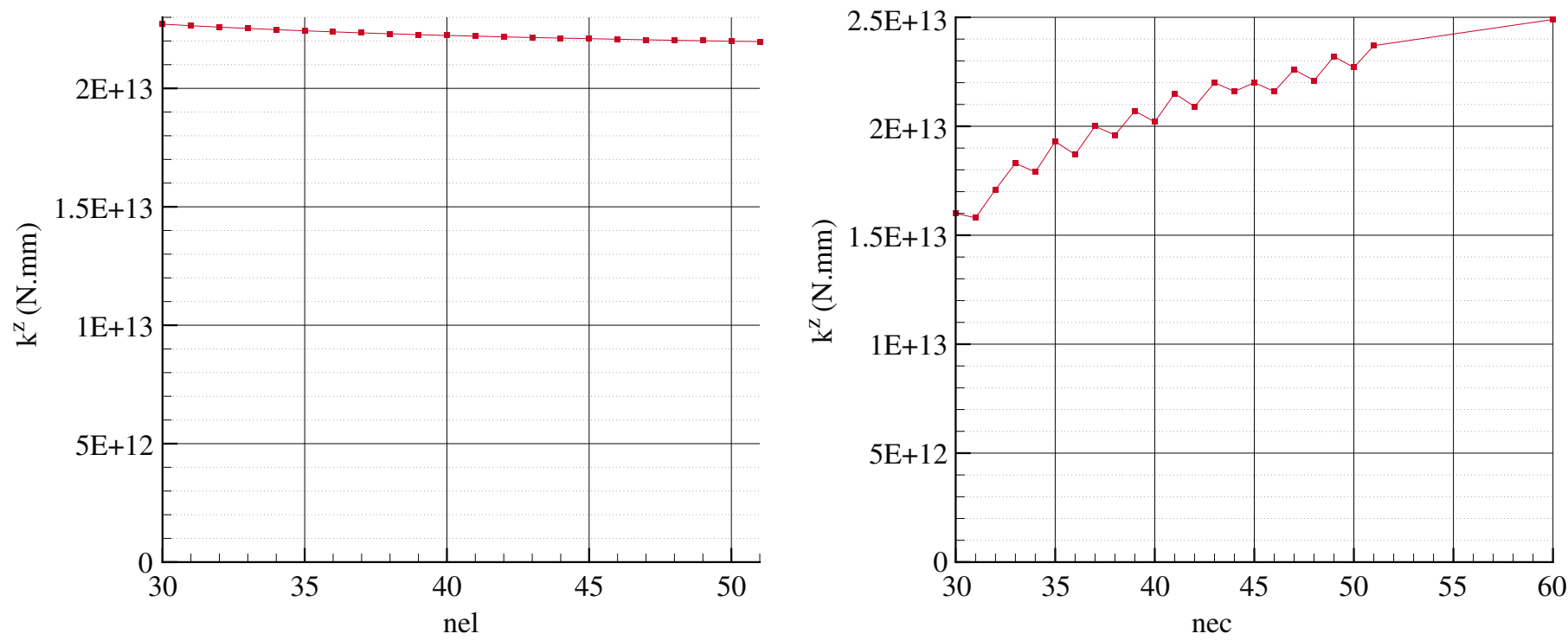

Figure 6. Stiffness $k^{z}$ versus element numbers nel and nec.

which are retained henceforth

$$
\begin{aligned}
& e_{\mathrm{th}}^{y}=\frac{-\left(D_{\mathrm{e}}^{3}-D_{\mathrm{i}}^{3}\right)}{3\left(D_{\mathrm{e}}^{2}-D_{\mathrm{i}}^{2}\right)} \frac{\cos \psi \sin \varphi}{\pi-\varphi} \\
& e_{\mathrm{th}}^{z}=\frac{-\left(D_{\mathrm{e}}^{3}-D_{\mathrm{i}}^{3}\right)}{3\left(D_{\mathrm{e}}^{2}-D_{\mathrm{i}}^{2}\right)} \frac{\sin \psi \sin \varphi}{\pi-\varphi}
\end{aligned}
$$

Figure 6 also shows that $k^{z}$ varies little with nel while it almost doubles as nec goes from 30 to 200 . 

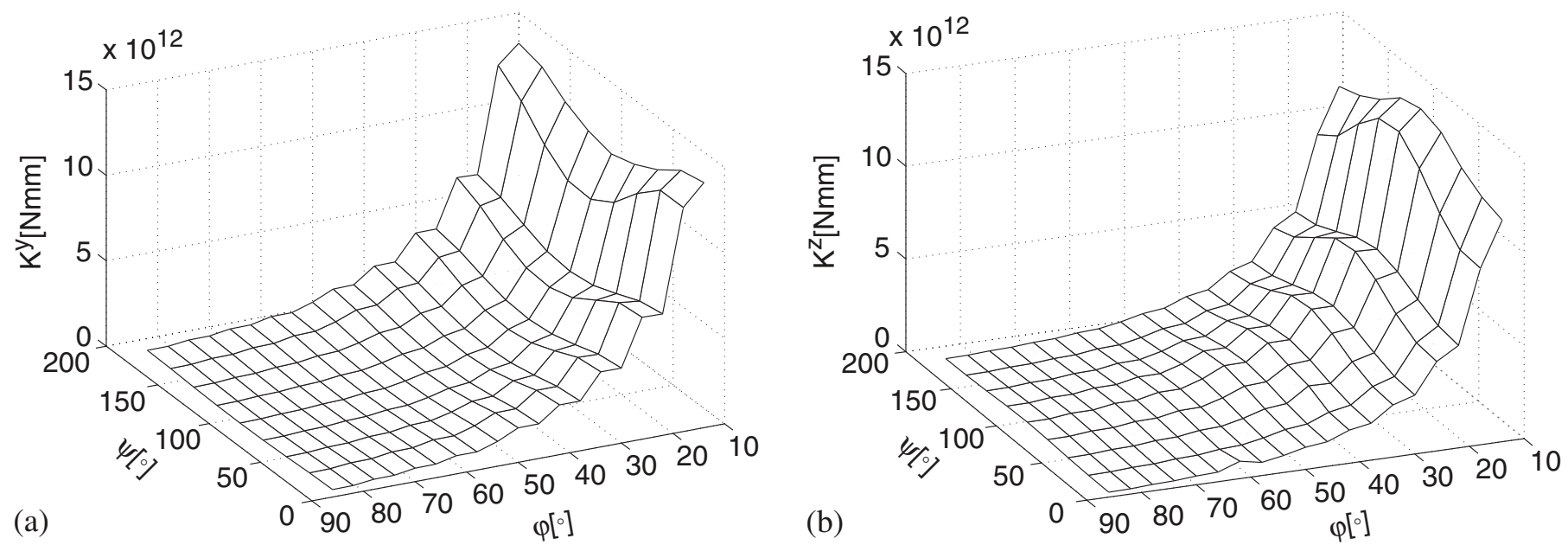

Figure 7. Surfaces of (a) $k^{y}$ and (b) $k^{z}$ for $\lambda=90^{\circ}$.
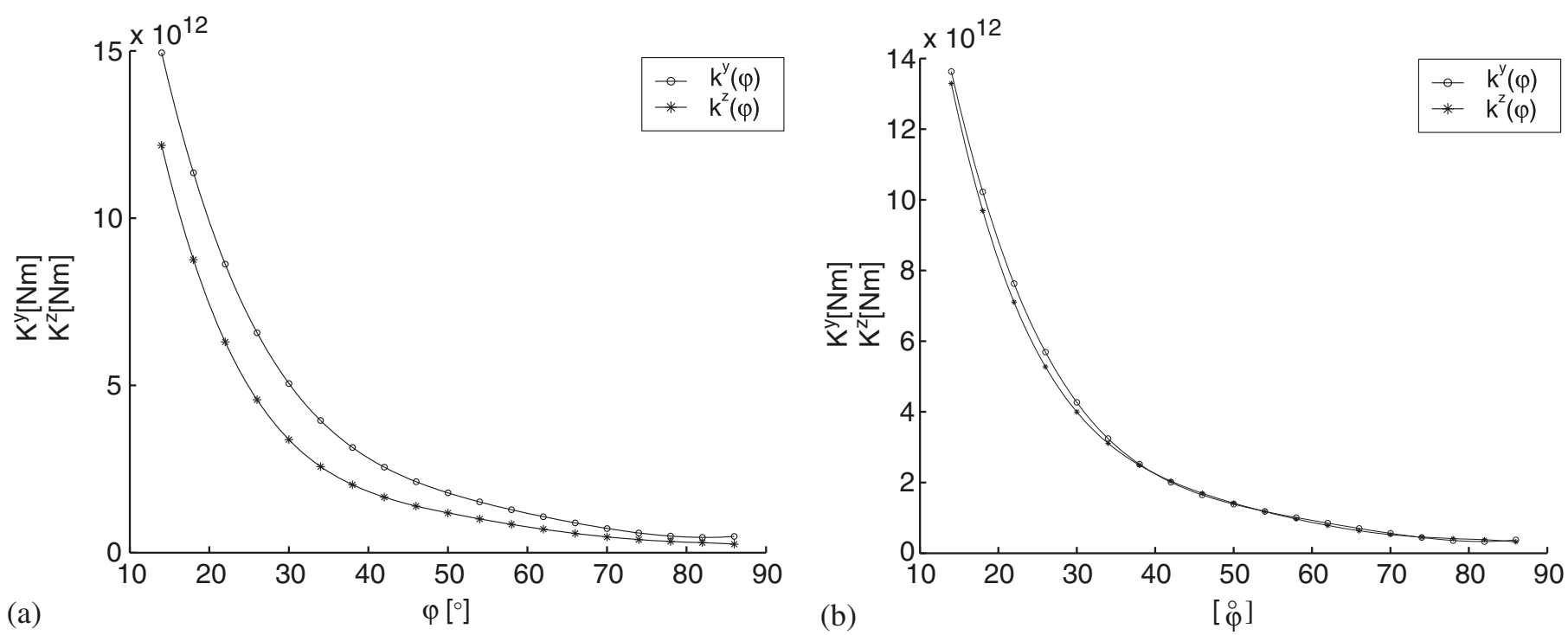

Figure 8. Curves of $k^{y}$ and $k^{z}$ for (a) $\psi=0^{\circ}$ and (b) $\psi=45^{\circ}$, with $\lambda=90^{\circ}$.

For specific studies where geometry is fixed, we have selected 50 and 110, respectively, for nel and nec. This choice was confirmed by a sensitivity study concerning geometrical parameters $\varphi$, $\psi$ and $\lambda$.

In the objective to give an overview on the main trends which governs parameters variations, we select in the following nel $=20$ and nec $=50$. It allows to reduce widely the number of calculations.

\subsection{Results: the identified model parameters}

A series of computations for identifying $k^{y}$ and $k^{z}$ are undertaken by considering various geometry values $\left(\lambda, \psi\right.$ and $\varphi$ ). Figures 7(a) and (b) depict the surfaces of $k^{y}$ and $k^{z}$ versus $\varphi$ and $\psi$, for $\lambda$ fixed to $90^{\circ}$ (the tube then is perpendicular to the plane). For a fixed $\varphi$ value, $k^{y}$ reaches two local maxima at $\psi=0^{\circ}$ and $180^{\circ}$ and one local minimum at $\psi=90^{\circ}$; this is most noticeable for small $\varphi$ values. On the other hand, $k^{z}$ presents an opposite variation, with two local minima and one local maximum. These variations can be accounted for by simple geometrical considerations. 
Indeed, for a given $\varphi$, the larger $\psi \in\left[0^{\circ}, 90^{\circ}\right]$, the more so is the projected area of the crack on the $y$-axis and the smaller the projected area on $z$-axis; hence, the inertia and stiffness of the section with respect to the $y$-axis increase whereas those with respect to the $z$-axis decrease. The opposite trend occurs for $\psi \in\left[90^{\circ}, 180^{\circ}\right]$.

Figure 8(a) shows the curves of $k^{y}$ and $k^{z}$ for $\lambda=90^{\circ}$ and $\psi=0^{\circ}$. They are obtained by fitting all the numerical results presented in Figure 7 using a polynomial function of degree 5. In this case, stiffness $k^{y}$ is higher than $k^{z}$ and the difference between the two values decreases as $\varphi$ increases; this is due to the fact that the projections of the crack surface onto $y$ and $z$-axes become closer as $\varphi$ increases. In the particular case when $\lambda=90^{\circ}$ and $\psi=45^{\circ}$, the projections of the crack surface are identical and so are stiffnesses $k^{y}$ and $k^{z}$ for the cracked surface, as clearly shown in Figure $8(\mathrm{~b})$. The difference between the unfitted and fitted curves for the last case reaches $6.9 \%$ for small crack openings.

\section{APPLICATION}

A computer program has been developed on the basis of the previous analysis to deal with framed structures. It includes the proposed CBFE together with classical beam elements to model uncracked tubes and plate elements with rectangular sections.

By means of this program, the CBFE model is assessed through the example of a cracked tube welded to a flexible plate (Figure 9). The tube is of length $l=10.2 \mathrm{~m}$, external diameter $D_{\mathrm{e}}=1.12 \mathrm{~m}$ and internal diameter $D_{\mathrm{i}}=1.08 \mathrm{~m}$. The plate is rectangular, twice as long as the tube; its section is rectangular of width $a=2.2 \mathrm{~m}$ and thickness $b=0.23 \mathrm{~m}$. The tube is welded perpendicularly to the plate at the middle of the plate. It is assumed that the symmetry plane of the crack is the same as that of the structure, i.e. $\lambda=90^{\circ}, \psi=0^{\circ}$. The tube is subjected to the force $F=-10^{6} \mathrm{~N}$ applied at its free end and directed along $y$-axis parallel to the plate length. The results are compared with (i) those from the strength of materials and (ii) those from 3D finite element computations using 20-node brick elements as shown in Figure 9.

(a)

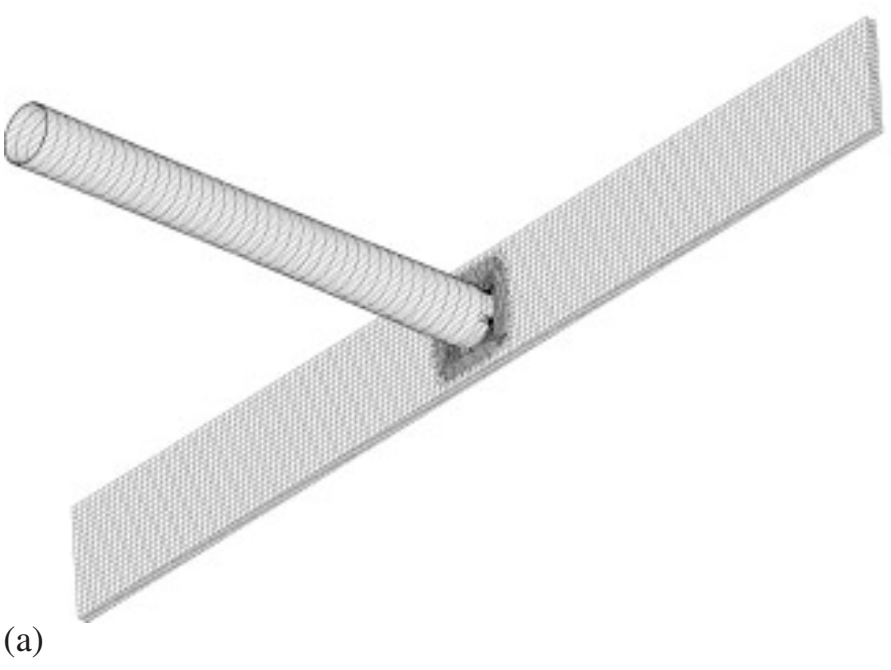

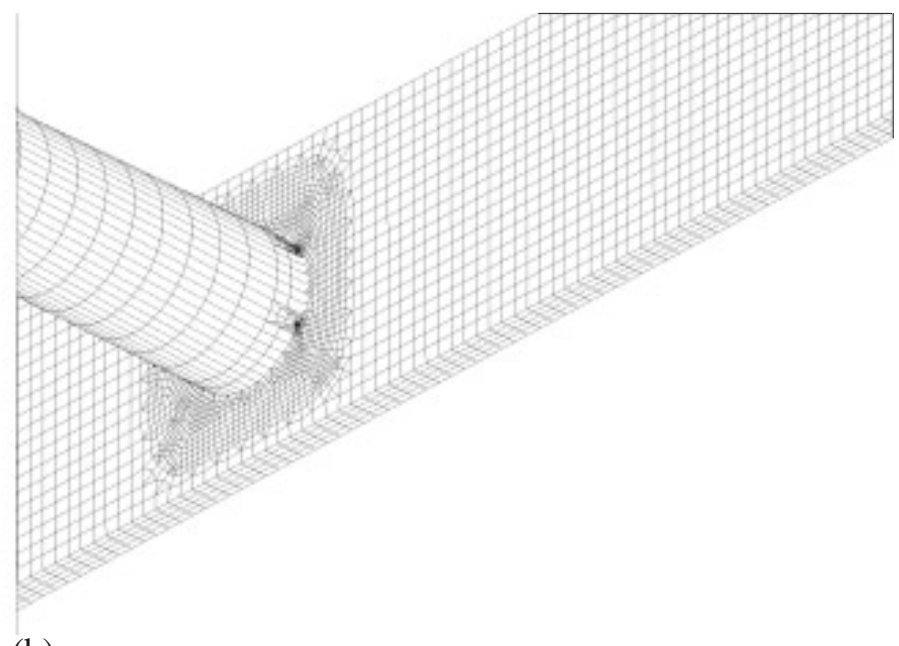

(b)

Figure 9. Mesh of the cracked tube welded to the plate: (a) mesh of the whole structure and (b) zoom of the cracked region. 


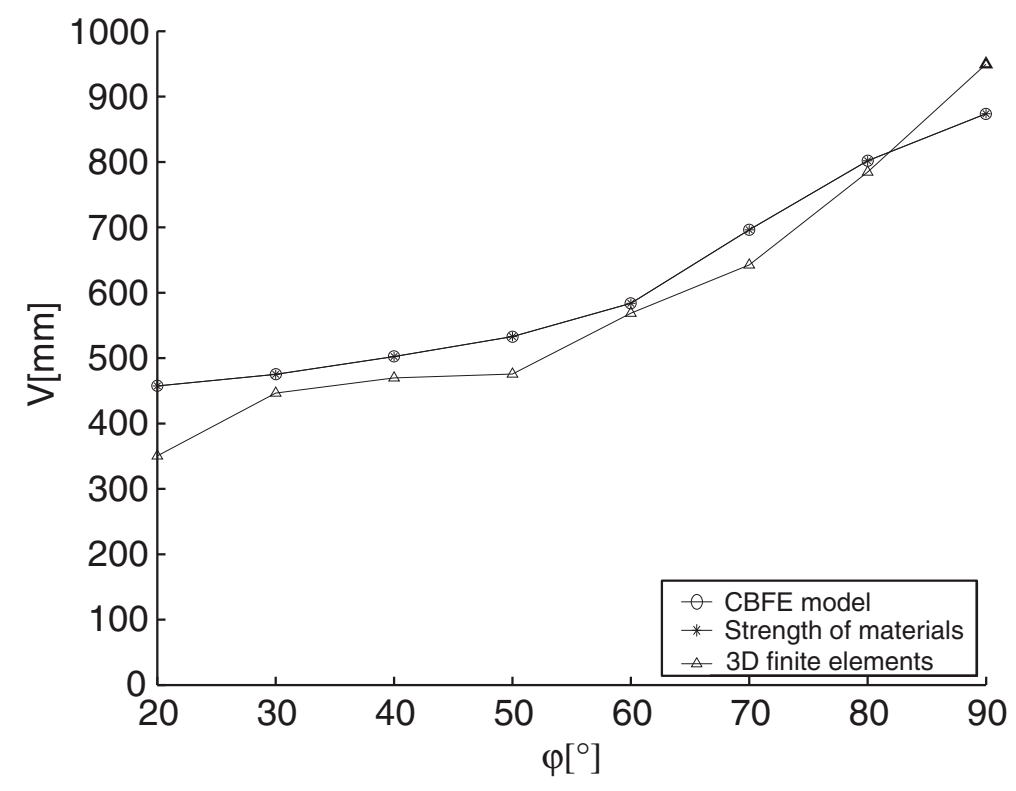

Figure 10. Deflection at the free end of the cracked tube.

Table I. Deflection at the free end by the three methods: CBFE model, strength of materials and 3D finite elements.

\begin{tabular}{lcccccccc}
\hline$\varphi$ & $20^{\circ}$ & $30^{\circ}$ & $40^{\circ}$ & $50^{\circ}$ & $60^{\circ}$ & $70^{\circ}$ & $80^{\circ}$ & $90^{\circ}$ \\
\hline CBFE model (mm) & 457.5 & 475.1 & 502.4 & 533 & 583.8 & 696.2 & 801.8 & 873.8 \\
Strength of materials (mm) & 457.5 & 475 & 502.3 & 532.9 & 583.8 & 696.2 & 801.7 & 873.7 \\
3D finite elements (mm) & 350.4 & 446.5 & 469.7 & 475.7 & 568.7 & 642.6 & 784.5 & 949.6 \\
Difference CBFE/3D FE (\%) & 23 & 6 & 7 & 11 & 3 & 8 & 2 & 8 \\
\hline
\end{tabular}

The deflection can be readily derived from the strength of the materials, to do this one just has to carefully distinguish the second moment of area for a tube section $I_{\text {tube }}=\pi\left(D_{\mathrm{e}}^{4}-D_{\mathrm{i}}^{4}\right) / 64$ from that for the plate section $I_{\text {plate }} a b^{3} / 12$. One then obtains

$$
v-\frac{F l^{3}}{3 E I_{\text {tube }}}-\frac{F l^{3}}{8 E I_{\text {plate }}}-\frac{F l^{2}}{k^{z}}
$$

where $E=2.1 \times 10^{11} \mathrm{~N} / \mathrm{m}^{2}$ is the Young modulus and $k^{z}$ the spring stiffness from the cracked beam model.

Figure 10 and Table I show the deflection $v$ at the tube free end versus the half-opening $\varphi$ of the crack, obtained from the cracked beam model CBFE, the 3D finite elements and the strength of materials. The cracked beam model and the strength of the materials give almost identical results. The difference between 3D finite element results and the two other theories reaches $23 \%$ for $\varphi=20^{\circ}$, yet it does not exceed $11 \%$ for other $\varphi$ values. In fact, the $23 \%$ difference should not be taken into account, since other computations which are not reported here show that it is difficult to obtain optimal 3D meshes and good results for small crack opening values, say less or equal to $20^{\circ}$. 


\section{CONCLUSIONS}

A new finite element has been presented in order to take into account the effect of large throughcracks in the structural analysis of steel-framed structures like the jacket offshore platforms. This cracked beam element based on the strain energy involves four parameters (two eccentricities and two stiffnesses) which represent the loss of stiffness. These parameters have been identified on the basis of several joint configurations. Then, the cracked element model has been applied on a T-tubular joint, giving results which are in very good agreement with the strength of materials, and quite satisfactory agreement with $3 \mathrm{D}$ finite elements.

\section{REFERENCES}

1. Rouhan A. Évaluation de l'intégrité structurale des plates-formes offshore existantes, à partir de données d'inspections (Assessment for the structural integrity of existing offshore platforms). Ph.D. Thesis, University in Nantes, France, November 2001.

2. Rouhan A, Wielgosz C. A beam finite element for through-cracked tubular node behavior modelling. Communications in Numerical Methods in Engineering 2002; 18:555-564.

3. Morin G. Requalification des structures offshores existantes: modes de ruines (Reassessment for existing offshore structures: study of collapse modes). Principia Recherche Developpement S.A., France.

4. Mohamed A. Modèle mécano-fiabiliste linéarisé pour l'analyse des structures. Application aux plates-formes marines (A linearized reliability model for mechanical structure analysis with application to marine platforms). Ph.D. Thesis, University Blaise Pascal, France, February 1993.

5. Pedersen C, Madsen HO, Nielsen JA, Riber JP, Krenk S. Reliability based inspection planning for the tyra field. The 11th International Conference on Offshore Mechanics and Arctic Engineering, Calgary, Alberta, Canada, 7-12 June 1992; 255-263.

6. Tieyun C, Huiyuan Z. Local joint flexibility multibrace tubular joints. China Ocean Engineering 1993; 7(2): 125-138.

7. Buitrago J, Healy BE, Chang TY. Local joint flexibility of tubular joints, OMAE, 1993.

8. Chakrabarti P, Mukkamala A, Abu I. Effect of joint behaviors on the reassessment of fixed offshore platforms in the bay of Campeche, Mexico. Odeh, OMAE, 2005-67052.

9. Chen T-Y, Zhang H-Y. Stress analysis of spatial frames with consideration of local flexibility of multiplanar tubular joint. Engineering Structures 1996; 18(6):465-471.

10. Lixian Y, Tieyun C, Shuiyun W. Local flexibility behavior of tubular joints and its effect on global analysis of tubular structures. China Ocean Engineering 1990; 4(4):371-384.

11. Matlab ${ }^{\circledR}$, release 7, ${ }^{\circledR} 1994-2006$ by The MathWorks Inc., 3 Apple Hill Drive, Natick, MA 01760-2098, U.S.A, info@mathworks.com www.mathworks.com

12. CAST3M, Finite Element Software, DEN/SAC/DM2S/SEMT_CEA/SACLAY—91191 Gif-sur-Yvette cedex, FRANCE, release 2004, www-cast3m.cea.fr 